\title{
The Effect of Ball Waviness on Nonlinear Vibration Associated with Rolling Element Bearings
}

\author{
S. P. Harsha and C. Nataraj \\ Center of Nonlinear Dynamics and Control, Villanova University, Villanova, PA-19085, USA
}

\section{P. K. Kankar}

Mechanical Engineering Department, CTAE, MPUAT-Udaipur, India

(Received 13 January 2004; revised 19 October 2005; accepted 10 March 2006)

\begin{abstract}
An analytical model was developed to investigate the nonlinear vibrations of a rotor bearing system due to ball waviness. In the analytical formulation the contacts between the balls and the races are modelled as nonlinear springs, whose stiffnesses are obtained by using Hertzian elastic contact deformation theory. The governing differential equations of motion are obtained by using Lagrange's equations. The implicit type of numerical integration technique Newmark- $\beta$ with Newton-Raphson method is used to solve the nonlinear differential equations iteratively. A computer program was developed to simulate the effect of ball waviness. The formulation predicts the discrete spectra with specific frequency components for each order of ball waviness. Numerical results obtained from the simulation are compared with those of prior researchers.
\end{abstract}

\section{Nomenclature}

$F_{u} \quad$ - unbalance force, $\mathrm{N}$

I $\quad-$ moment of inertia of each rolling element

Irotor - moment of inertia of the rotor

$I_{\text {in }} \quad-$ moment of inertia of the inner race

$I_{\text {out }} \quad-$ moment of inertia of the outer race

$k-\quad-$ waviness order

$K-$ constant of proportionality, $\mathrm{N} / \mathrm{mm}^{3 / 2}$

$L \quad$ - arc length, mm

$M_{\text {rotor }} \quad-$ mass of the rotor, $\mathrm{kg}$

$m_{\text {in }} \quad-$ mass of the inner race, $\mathrm{kg}$

$m_{j} \quad-$ mass of the rolling elements, $\mathrm{kg}$

$m_{\text {out }} \quad-$ mass of the outer race, $\mathrm{kg}$

$N_{w} \quad$ - number of wave lobes

$N_{b} \quad-$ number of balls

$p \quad-$ empirical constant for a particular geometry

$q \quad$ - empirical constant for a particular geometry

$R \quad$ - radius of outer race, $\mathrm{mm}$

$r \quad-$ radius of inner race, $\mathrm{mm}$

$r_{\text {in }} \quad-$ position of mass centre of inner race

$r_{p u t}-$ position of mass centre of outer race

$T \quad-$ kinetic energy of the bearing system

$T_{\text {rotor }}-$ kinetic energy of the rotor

$T_{i \text { race }}-$ kinetic energy of the inner race

$T_{o_{-} \text {race }}-$ kinetic energy of the outer race

$T_{\text {roll_e }}-$ kinetic energy of the rolling elements

$V \quad-$ potential energy of the bearing system

$V_{\text {shaft }} \quad$ - potential energy of the shaft

$V_{\text {i_race }}$ - potential energy of the inner race

$V_{\text {o_race }}-$ potential energy of the outer race

$V_{\text {roll_e }}-$ potential energy of the rolling elements

$V_{\text {spring }}$ - potential energy of the springs

$x_{i n}, y_{\text {in }}-$ centre of inner race

$x_{\text {out }}, y_{\text {out }}-$ centre of outer race $\delta-$ deformation at the point of contact at inner and outer race, $\mathrm{mm}$

$(\dot{\phi})_{\text {in }} \quad$ - angular velocity of inner race

$(\dot{\phi})_{\text {out }} \quad$ - angular velocity of outer race

$\omega_{b p} \quad$ - ball passage frequency, $\mathrm{Hz}$

$\omega_{w p} \quad-$ wave passage frequency, $\mathrm{Hz}$

$\left(\Pi_{i}\right)_{b} \quad$ - amplitude of the wave at ball, $\mu \mathrm{m}$

$\rho_{j} \quad-$ radial position of the rolling element

$\rho_{r} \quad-$ radius of each rolling element

$\theta_{j} \quad-$ angular position of rolling element

$\chi_{j} \quad-$ position of $j$-th rolling element from the centre of inner race

FFT - Fast Fourier Transformation

BPF - Ball Passage Frequency, Hz

BPV - Ball Passage Vibration, $\mathrm{Hz}$

WPF - Wave Passage Frequency, $\mathrm{Hz}$

\section{INTRODUCTION}

Rolling bearings are the most used components in machinery and are employed in a wide variety of rotating machinery from small handheld devices to heavy duty industrial systems. It is generally known that ball bearings cause vibrations even under ideal conditions; ${ }^{\mathbf{1}, 2}$ furthermore, in the presence of defects, which are naturally introduced due to manufacturing limitations and operational conditions, the vibrations and noise produced can be substantially complex and quite difficult to analyse.,

In addition to the fact that most machines are nonlinear devices with very complicated time signatures, these bearing defects tend to introduce strong nonlinearities. Hence, standard linear techniques that are employed widely in industry are incapable of predicting their response accurately. In addition, since the mathematical underpinnings of linear and non- 